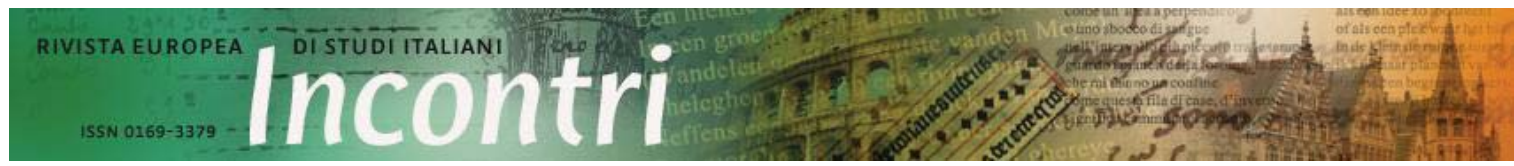

URN:NBN: NL:UI:10-1-114270 - Publisher: Igitur publishing

Content is licensed under a Creative Commons Attribution 3.0 License

Anno 28, 2013 / Fascicolo 2 - Website: www.rivista-incontri.nl

\title{
La rappresentazione di un potere Il vescovo di Anagni Pietro da Salerno († 1105)
}

\section{Cristiano Mengarelli}

Nell'ambito della storia di Anagni, la figura del vescovo Pietro da Salerno ha rivestito un ruolo fondamentale per l'affermazione del peso politico che la cittadina ebbe nel corso dell'intero medioevo; ${ }^{1}$ il suo operato come pastore d'anime, i suoi interventi nel quadro urbano ed il riflesso di questo insieme di elementi nella memoria storica formatasi attorno al vescovo contribuiranno a consolidarne la tradizione storica. II suo magistero episcopale venne attuato nella convinzione di agire in funzione di un potere acquisito, da consolidare nel suo tempo, e da offrire come eredità stabile e consolidata per il futuro.

Le modalità di rappresentazione di questo potere hanno riguardato un programma di azioni, incentrate sul rinnovamento urbanistico di un intero quartiere cittadino, funzionale alla creazione di un 'macro-palinsesto' architettonico che potesse servire da scenografia per un manifesto politico fondato sul recupero, attraverso la continuità, delle memorie storiche, agiografiche e materiali che dovevano definire e richiamare, attraverso linguaggi semplici ma di impatto immediato, la dimensione del potere vescovile esercitato sulla città di Anagni. Pertanto siamo al cospetto della legittimazione di un potere attraverso la rappresentazione di significati e rimandi storici, realizzata comunque nel solco stilistico della tradizione culturale del suo tempo.

In questo senso la figura di Pietro costituisce il fulcro di un insieme di realtà che hanno concorso a dare una immagine, una rappresentazione quindi, del suo ruolo; di cui, in questa occasione, se ne possono cogliere, a fronte di problematiche complesse e documentazioni lacunose, solo alcuni aspetti singoli, frutto dell'osservazione delle testimonianze materiali che non della sola tradizione storico-agiografica, fino ad ora predominante negli studi su Pietro da Salerno. La scelta di destinare un personaggio come Pietro per la sede anagnina tradisce chiaramente l'intenzione di affidare ad una guida sicura una diocesi considerata fondamentale per gli equilibri della regione. Non a caso, per esempio, nel corso della seconda metà dell'XI secolo la diocesi di Anagni espande il suo dominio territoriale inglobando la vicina diocesi di Trevi, la cui importanza strategica era evidente, quale sede del ricco santuario della SS. Trinità di

\footnotetext{
${ }^{1}$ II presente contributo costituisce una estrema sintesi dei diversi studi condotti sul medioevo anagnino in ambito universitario, sviluppati da ultimo in C. Mengarelli, Scelte insediative ed organizzazione urbanistica nelle città del comprensorio dei Monti Ernici dalla tarda antichità al medioevo, tesi di dottorato Università di Roma Sapienza, giugno 2009; ed in parte confluiti in Idem, 'Le testimonianze storiche e materiali sulla Cattedrale di Anagni nell'Alto medioevo', in: Bollettino d'Arte, volume speciale, 2006, pp. 69-80.
} 
Monte Autore e soprattutto delle sorgenti dell'Aniene, da cui derivavano poi le fonti di alimentazione degli acquedotti romani. ${ }^{2}$

II nostro vescovo, esponente della nobiltà salernitana, svolse la sua formazione monastica presso il S. Benedetto a Salerno, ${ }^{3}$ dove le sue capacità, e le doti anche in chiave culturale, vennero notate da Ildebrando di Sovana, che, stando al raccolto agiografico, volle coinvolgere il giovane monaco nell'amministrazione ecclesiastica. ${ }^{4}$ Pietro divenne vescovo di Anagni nel corso degli anni Sessanta dell' XI secolo. II primo intervento che possiamo ricordare, sulla base della documentazione notarile, ${ }^{5}$ fu quello rivolto a riorganizzare le pertinenze fondiarie dell'episcopio, con un riordino delle proprietà ecclesiastiche, anche in ragione della necessità di acquisire spazio edificabile attorno alla vecchia cattedrale altomedievale, nell'ottica di avviare quel percorso che porterà alla definizione topografica del quartiere episcopale. ${ }^{6}$ Un primo passo dunque a cui doveva far seguito quello più impegnativo: la costruzione di un nuovo edificio successivo all'abbattimento della vecchia Cattedrale, di cui ne avrebbe comunque conservato I'intitolazione alla Vergine (Fig. 1). Questo dettaglio denota I'intenzione di perseguire da subito un obbiettivo preciso: fornire la sua sede di un complesso architettonico e topografico capace da solo di rappresentarne la grandezza ed implicitamente la stessa potenza del magistero episcopale, attraverso la sua ramificazione materiale nel tessuto cittadino (Fig. 2). Sebbene la scelta topografica fosse già segnata a favore dell'altura più rilevante tra quelle che caratterizzano il quadro urbano anagnino, non di meno le motivazioni dovevano risultare fortemente sentite. ${ }^{7}$ Dal vecchio edificio altomedievale vennero salvati un gran numero di arredi scultorei del tipo ad intreccio vimineo, reimpiegati poi nella decorazione della struttura romanica, in particolare nelle ghiere degli archetti ciechi e delle finestre che decorano la parte occidentale del complesso (Fig. 3), ma anche lungo la facciata dell'edificio,

\footnotetext{
2 F. Caraffa, “"Treba Augusta” (Trevi nel Lazio) alle sorgenti dell'Aniene, municipio romano e sede episcopale', in: Bollettino della sezione per il Lazio meridionale della Società romana di Storia Patria I (1951), pp. 77-88. Il passaggio progressivo della diocesi di Trevi verso I'annessione con I'episcopato anagnino è avvenuto tra i pontificati di Nicola II ed Urbano II, con motivazioni addotte a riguardo della povertà della diocesi e la scarsità degli abitanti. A questa decisione fece seguito un lungo contenzioso conclusosi solo nel XIII secolo con una sorta di patteggiamento economico calibrato sulle entrate del santuario di Vallepietra.

${ }^{3}$ L'abbazia di S. Benedetto era contigua al Castel Terracena, cioè il centro di potere della compagine normanna a Salerno (vedi A. R. Amarotta, Salerno romana e medievale. Dinamica di un insediamento, Salerno, P. Laveglia, 1989, pp. 99-113), ed alla chiesa, poi divenuta monastero, di S. Michele, per la quale vedi A. Finella, Storia urbanistica di Salerno nel medioevo, Roma, Bonsignori, 2005, pp. 68-69.

${ }^{4}$ Bibliotheca Apostolica Vaticana, Codice Chigiano C. VIII. 235, Lectionarium per annum et proprium et comune de Sanctis ad usum Ecclesiê Anagninae, ff. 195-197; cfr. R. Grégoire, 'La memoria agiografica liturgica di Pietro da Salerno', in: Pietro da Salerno (†1105) monaco benedettino e vescovo di Anagni, a cura di L. Cappelletti \& A. Molle, Venafro, Edizioni Eva, 2006, pp. 17-64. Cfr. anche G. M. Cantarella, Il sole e la luna. La rivoluzione di Gregorio VII papa 1073-1085, Roma-Bari, Laterza, 2005, pp. 56-57; 66-67 sulla esigenza sentita tra i riformatori di promuovere nei ranghi ecclesiastici personaggi culturalmente preparati.

${ }^{5}$ Ancora valida la sintesi in R. Ambrosi de Magistris, Storia di Anagni, vol. I, Roma, Roma laziale tipografica editrice, 1889 , p. 342.

${ }^{6}$ L. Pani Ermini, 'Renovatium murorum. Tra programma urbanistico e restauro conservativo: Roma ed il Ducato romano', in: Committenti e produzione artistico-letteraria nell'alto medioevo occidentale, XXXIX Settimana di studio del CISAM, Tomo II, Spoleto, Centro Italiano di Studi alto medievali, 1992, pp. 485-530 (in particolare p. 530).

${ }^{7}$ Per riflettere su come poteva essere percepita a livello simbolico la presenza di un complesso episcopale sull'altura di una città, occorre ricordare il passo di Matteo 5, 14: 'Voi siete la luce del mondo, la città posta sopra un monte non può essere nascosta'.
} 
realizzata nella prima fase costruttiva della Cattedrale. ${ }^{8}$ La posizione dei fregi alto medievali riutilizzati in facciata (Figg. 4-5), se davvero presenti fin dall'origine per intero, come sembra assai probabile, ${ }^{9}$ e dei frammenti di fregio di epoca romana, è piuttosto ordinata, con un taglio delle lastre e la scansione metrica dei singoli blocchi accurata. ${ }^{10}$ Tra i materiali figurativi di epoca alto medievale, venne reimpiegata, di fianco al portale d'ingresso sul lato destro, un epigrafe in cui è citato il vescovo Romualdo (Fig. 5), colui a cui si tende ad attribuire l'impianto della struttura alto medievale abbattuta nell'XI secolo. ${ }^{11}$

Va precisato come nella Cattedrale di Pietro il recupero ed il riuso degli elementi storici non ha un valore di praticità funzionale: I'edificio è un esempio di architettura interamente realizzata in materiale omogeneo con cortina di calcare locale, tagliato in blocchi quadrati di ottima fattura, messi in opera con apparecchi regolari. La scelta di recuperare materiali di epoca altomedievale se non appare certo originale, ${ }^{12}$ sembra però indirizzata verso una lettura funzionale per una rappresentazione di significati. ${ }^{13}$ Lungo i pilastri-lesena della facciata vennero impiegati anche delle lastre prelevate da un monumento funerario romano del tipo con decorazione a 'fregio dorico', ed inserite alla terminazione del pilastro in un gioco decorativo che rappresentava un'alternativa stilistica alla tradizionale presenza del capitello (Figg. 4-5). Questo utilizzo dell'antico rimane circoscritto ad un richiamo sobrio alla romanità, che non ostenta quindi la monumentalità decorativa dei frammenti scelti da Alfano per la Cattedrale di Salerno, ${ }^{14}$ né di quella ostentata, almeno nelle fonti, da Desiderio per l'Abbazia di Montecassino, ${ }^{15}$ ed ancor meno di quella più grossolana richiamata per la corte normanna. ${ }^{16}$

La tipologia decorativa 'a fregio dorico' doveva apparire come un segno distintivo di un richiamo all'antico, in cui si rivendicava una discendenza storica nel solco di una

\footnotetext{
${ }^{8}$ G. Carbonara, lussu Desiderii. Montecassino e l'architettura campano-abruzzese nell'undicesimo secolo, Roma, Università degli Studi di Roma-Istituto di Fondamenti dell'Architettura, 1979, p. 130.

${ }^{9}$ V. Piacentini, 'La Cattedrale di Anagni e il suo contesto urbano', in: Bollettino d'Arte, cit., pp. 135-157, a p. 150 pubblica un rilievo della prima metà del XIX secolo in cui la rappresentazione grafica della facciata non sembra presentare tutta la teoria delle lastre altomedievali. L'edificio subì dei restauri tra il XIX e XX secolo, tra i quali però non si fa mai alcun accenno al riassetto della facciata, cfr. A. Ricci, 'La Cattedrale di Anagni. Il percorso degli interventi operati dall'anno della sua fondazione fino ai nostri giorni', in: Bollettino d'Arte, cit., pp. 297-304. L'osservazione diretta conferma come le lastre altomedievali appaiono in fase con la realizzazione del portale originario (Fig. 6).

10 Vedi A. Esch, S.v. 'Reimpiego', in: Enciclopedia dell'arte medievale, vol. IX, Roma, Istituto dell'Enciclopedia Italiana, 1998, pp. 876-883, in particolare pp. 887-888.

11 Mengarelli, 'Le testimonianze storiche', cit., p. 75.

12 D. Fiorani, 'Costruire recuperare e rifinire. Tecniche edilizie basso medievali nel centro Italia', in: II reimpiego in architettura. Recupero, trasformazione, uso, a cura di J.-F. Bernard, P. Bernardi \& D. Esposito, Roma, École française de Rome, 2008, pp. 575-589.

${ }^{13}$ V. Pace, 'La Riforma e i suoi programmi figurativi: il caso romano, fra realtà storica e mito storiografico', in: Roma e la riforma gregoriana. Tradizioni e innovazioni artistiche (XI-XII secolo), a cura di S. Romano \& J. Enckell J ulliard, Roma, Viella, 2007, pp. 49-59.

${ }^{14} \mathrm{Cfr}$. V. Pace, 'La Cattedrale di Salerno. Committenza programma e valenze ideologiche di un monumento di fine XI secolo nell'Italia meridionale', in: Desiderio di Montecassino e l'arte della Riforma Gregoriana, a cura di F. Avagliano, Montecassino, Pubblicazioni Cassinesi, 1997, pp. 189-230.

${ }^{15}$ L. de Lachenal, Spolia. Uso e reimpiego dell'antico dal III al XIV secolo, Milano, Longanesi, 1995, pp. 158170 .

${ }^{16} \mathrm{Cfr}$. L. De Lachenal, 'Reimpiego dell'antico e ideologia politica fra Roma e I'Italia Meridionale in età normanna: alcune osservazioni', in: Il passato riproposto. Continuità e recupero dall'antichità ad oggi, a cura di B.M. Giannattasio, Genova, Dipartimento di Archeologia, Filologia classica e loro tradizioni "Francesco della Corte", 1999, pp. 93-129.
} 
tradizione italica centro-meridionale, ${ }^{17}$ ben rappresentata dal coevo esempio del $\mathrm{S}$. Domenico di Sora, ${ }^{18}$ dove l'impiego di questo elemento è alquanto diffuso, ma in modo apparentemente più casuale. Ai lati del portale vennero anche collocate due lastre in travertino, di cui una era parte di un epigrafe monumentale; ${ }^{19}$ mentre la seconda reca incisa in caratteri capitali la sola lettera ' $P$ ' tracciata in posizione apparentemente casuale sull'ampia superficie del blocco.

II programma decorativo che aveva ornato il vecchio edificio del IX secolo, doveva aver già accompagnato la condivisione, da parte del vescovo Romualdo, delle linee dettate da Roma e rappresentate nello specifico anche dall'adesione verso le spinte agiografiche come quelle sostenute da papa Pasquale $\mathrm{I}^{20}$ di cui la figura di S. Potenziana ne è un esempio. ${ }^{21}$ Anche Romualdo era stato un monaco benedettino di origine longobarda, ${ }^{22}$ formatosi però nel cenobio sublacense. Caratteristiche che potevano essere perfettamente percepite da Pietro, anche con la sola lettura degli apparati epigrafici coevi al IX secolo, e nelle quali il vescovo di origine salernitana poteva rispecchiare anche la sua situazione.

La sua fedeltà a Roma, ribadita anche grazie al recupero della tradizione locale altomedievale, forniva una legittimazione della sua posizione, consolidando quindi quel processo di formazione di un tassello fondamentale del continuum di un percorso storico e politico ben chiaro: il passato salvato dall'oblio, a cui sarebbe stato destinato in ragione dell'intervento petrino, è direttamente quello del papato cosiddetto 'carolingio', un momento 'fondativo' di per sé del percorso storico intrapreso dalla stessa chiesa romana fino ad allora. ${ }^{23}$ La scelta di apporre l'epigrafe di Romualdo vuole rappresentare un concetto molto semplice: I'operato di Pietro è anche un 'rifacimento', per quanto monumentale, di una precedente fondazione. Purtroppo I'assenza in situ dell'epigrafe petrina di fondazione, non agevola nello sviluppare ulteriormente questo discorso. ${ }^{24}$

\footnotetext{
${ }^{17}$ Cfr. C. Capaldi, Severo more doricum. Espressioni del linguaggio figurativo augusteo in fregi dorici della Campania, Pozzuoli, Naus, 2005, p. 106 con bibliografia. II monumento a fregio dorico ebbe un'ampia diffusione nel corso della tarda età repubblicana, in un momento in cui I'Italia stava compiendo quel processo di romanizzazione attuato anche con soluzioni, come quelle delle guerre civili, che stravolsero in modo netto il rapporto tra Roma ed i maggiorenti locali arrivando ad esigerne la totale adesione. In questo senso quell'espressione figurativa era divenuta sinonimo dell'adesione alla causa di Roma; probabilmente questo nesso venne tramandato anche in età medievale, come un tratto distintivo per riconoscersi eredi della romanità. Vedi S. Settis, 'Continuità, distanza e conoscenza. Tre usi dell'antico. L'uso dell'antico nel Medioevo', in: Memoria dell'antico nell'Arte Italiana, a cura di S. Settis, vol. III, Torino, Einaudi, 1986, pp. 375-486, in particolare p. 403.

${ }_{18}$ Per la struttura cfr. Carbonara, lussu Desiderii, cit., p. 131. È importante sottolineare come lo stesso Domenico da Foligno provenisse da un ambiente storico-culturale quale quello umbro dove questi elementi decorativi erano patrimonio della tradizione romana, cfr. Capaldi, Severo more doricum, cit., p. 63.

${ }^{19} \mathrm{CIL} \mathrm{X}, 5954$

${ }^{20}$ Mengarelli, 'Le testimonianze storiche', cit., p. 69.

${ }^{21}$ Vedi ad esempio, Pace, 'La Cattedrale di Salerno', cit., p. 200; Pace, 'La Riforma', cit., pp. 53-55; S. Pudenziana nell'ambito della riforma doveva rappresentare qui valori legati alla verginità ed alla castità, che erano un passaggio-chiave del programma politico gregoriano, per cui vedi Cantarella, Il sole e la luna, cit., pp. 124-129.

22 Mengarelli, 'Le testimonianze storiche', cit., p. 69, n. 2.

${ }^{23}$ È opportuno ricordare come nell'XI secolo si ricercasse nel passato carolingio una giustificazione storica del potere imperiale, vedi Cantarella, Il sole e la luna, cit., pp. 187-188.

${ }^{24}$ Per la presenza dell'epigrafe di fondazione vedi un caso specifico: V. Pace, 'La sconfitta di un modello e del suo progettista: la Cattedrale di Aversa', in: Napoli Nobilissima, XXXIV (1995), pp. 123-129.
} 
La lunga e discussa gestazione della Cattedrale romanica successiva all'abbattimento della precedente fa supporre che vi sia stata la necessità di trasferire in un'altra sede lo svolgimento delle funzioni religiose, almeno per gli ultimi decenni dell'XI secolo. Questo dettaglio ci offre lo spunto per osservare la distribuzione delle presenze cultuali ragionevolmente ascrivibili a questo periodo nel quadro urbano (Fig. 1). Almeno dal IX secolo era sicuramente già esistente la chiesa di $\mathrm{S}$. Andrea, ${ }^{25}$ presso la quale è attestata anche una dedica a $\mathrm{S}$. Vito, in associazione con una struttura funzionalmente connessa con un ambiente interpretabile come cripta. La chiesa di S. Pancrazio è conosciuta invece dagli inizi dell' XI secolo, mentre è probabile che a cavallo del X secolo esistesse già la chiesa di S. Giovanni de Duce, posta nell'area del Foro antico, la cui titolatura sembra tradire la sua seriazione cronologica con un altro edificio dedicato a S. Giovanni. Infine una chiesa dedicata all'Arcangelo Michele, conosciuta come S. Angelo, pur essendo un'attestazione leggermente più tarda, presenta dei connotati che lasciano ipotizzare una sua pertinenza almeno all'XI secolo. ${ }^{26}$

Nei casi elencati possiamo ritrovare dei validi appoggi per guardare ad un intervento del vescovo in chiave urbana nel riqualificare e rinvigorire il quadro delle strutture con funzioni religiose. II richiamo alla tradizione cultuale di Salerno sembra insinuarsi nelle dediche a S. Vito o a S. Michele, ${ }^{27}$ di cui ad Anagni si conosce la presenza di un altro caso proprio nel tratto d'accesso alla collina del quartiere di 'Castello', dove Pietro prima, ed i vescovi ed i papi poi tra XII e XIII secolo, creeranno il cuore di quel complesso monumentale che è stata la città di Anagni nel tardo medioevo (Fig. 2). II richiamo a Salerno non è da vedersi solo come il desiderio di citare il proprio passato, ma anche come un segnale politico cioè l'immediatezza del riferimento alla personalità di Alfano, allora arcivescovo di Salerno, ed attraverso questi all'abate Desiderio di Montecassino. In questo senso andrebbe ascritta a tale fase storica la presenza ad Anagni di una dedica ai SS. Cosma e Damiano prossima all'area del palazzo cosiddetto di Bonifacio VIII, anch'esso già parte del complesso di edifici che definirono il nuovo quartiere episcopale (Figg. 1-2). La posizione della dedica ai santi Cosma e Damiano sembra per altro ricalcare quella pertinente all'omonima chiesa salernitana, ${ }^{28}$ in quel caso posizionata in prossimità dell'accesso dalla porta urbana che immetteva verso il quartiere della Cattedrale, mentre ad Anagni si trovava nel punto di accesso verso la collina su cui sorse il quartiere episcopale. Per i salernitani la traslazione delle reliquie dei due santi verso la Cattedrale di Salerno avvenuta sotto la reggenza di Alfano dovette costituire un momento solenne, il cui ricordo profondo verrà immortalato proprio in uno dei carmi composti dall'arcivescovo salernitano. ${ }^{29}$ Questa lettura d'altronde si inserirebbe nel medesimo solco interpretativo inerente la tipologia costruttiva della Cattedrale, per la quale la diretta ispirazione dal prototipo cassinese desideriano deve essere ribadito come avente un valore anche in chiave storico-politica. ${ }^{30}$

Nel campo della memoria cultuale l'impegno maggiore di Pietro fu riversato nel rapporto con la figura di S. Magno, su cui si è indirizzata una parte consistente della sua

\footnotetext{
${ }^{25}$ Mengarelli, 'Le testimonianze storiche', cit., p. 78, n. 11.

${ }^{26}$ Idem, 'Scelte insediative', cit., pp. 47-52.

${ }^{27}$ A Salerno sono attestati vari edifici dedicati a S. Vito, in parte già esistenti nell'XI secolo, cfr. Amarotta, Salerno romana e medievale, cit., pp. 272-273.

${ }^{28}$ Amarotta, Salerno romana e medievale, cit., p. 108.

${ }^{29}$ P. Delogu, Mito di una città meridionale (Salerno, secoli VIII-XI), Napoli, Liguori, 1977, p. 182.

${ }^{30}$ Carbonara, lussu Desiderii, cit., p. 130. Anche se è accertata l'esistenza di casi opposti, cioè di adesioni politiche senza una corrispettiva accettazione dei modelli culturali, per cui vedi Pace, 'La sconfitta di un modello', cit., pp. 126-127.
} 
'pastorale', quale ulteriore elemento di rappresentazione del suo ruolo. Come noto, le spoglie del santo arrivarono ad Anagni nel pieno IX secolo, dopo aver subito diversi spostamenti tra Fondi e Veroli; ${ }^{31}$ I'acquisizione di una nuova sede stabile per un culto così ambito era la conseguenza della potenzialità anagnina nel poter trattenere presso di sé queste preziose reliquie. Al ruolo che si stava consolidando per la diocesi di Anagni nel corso della prima metà del IX secolo mancava un tassello fondamentale, cioè la presenza di una robusta tradizione agiografica locale. II vescovo Romualdo, nella sua adesione al programma politico papale nel IX secolo doveva aver valorizzato apporti diretti dell'agiografia romana, a coprire un evidente lacuna, che rimarrà tale sostanzialmente fino all'XI secolo. L'agiografia anagnina contemplava la presenza di una figura minore quale era $\mathrm{S}$. Secondina, attestata però solo dal X secolo, ${ }^{32}$ la cui presunta origine locale è in realtà da connettere alla presenza ad Anagni dei monaci sublacensi nell'alto medioevo e forse andrebbe ricollegata anch'essa all'operato del vescovo Romualdo.

Pietro ha quindi incardinato il suo programma episcopale nel recuperare e rinvigorire il culto a S. Magno, sotto il cui patronato venne giustificata, come ricorda la tradizione agiografica, la responsabilità dell'impresa: la costruzione della nuova Cattedrale farà seguito all'apparizione in sogno a Pietro dello stesso S. Magno che indicherà al presule il luogo dove ritrovare le sue spoglie da cui partire per realizzare I'opera. ${ }^{33}$ Con la 'riscoperta' del culto e del luogo di sepoltura delle spoglie di S. Magno ad Anagni, Pietro operava anche un azione di ripristino delle motivazioni che avevano portato ad accogliere il santo ad Anagni in forma definitiva, cioè acquisire un patronato di rilievo, con un ampio bacino cultuale che investiva gran parte dell'Italia centromeridionale, ${ }^{34}$ a cui comunque riagganciare, come farà poi la tradizione agiografica già dal XII secolo, ${ }^{35}$ le scarse memorie 'locali' fino ad allora maturate. L'apporto materiale di Pietro fu notevole: il recupero storico della questione agiografica fu seguito da un riordino materiale, culminato nel raccogliere queste memorie nella nuova cripta, che divenne di fatto il sacrario dell'agiografica anagnina, ed in cui confluì, ovviamente, anche la memoria del vescovo salernitano dal XII secolo. La tradizione agiografica petrina ci informa che non sempre le sue scelte furono condivise dai chierici locali, e forse con loro anche da parte della popolazione anagnina. ${ }^{36}$ Le rimostranze, e le polemiche, dovettero costituire un dato incontrovertibile, se arrivarono a travalicare

\footnotetext{
${ }^{31}$ Da ultimo F. Carcione, 'S. Magno: problema agiografico e prospettive di ricerca in ambito aquinate', in: Magno di Trani. Memoria e culto di un martire paleocristiano nelle valli del Liri e del Sacco. Ricerche di agiografia-topografia-iconografia, a cura di F. Carcione, Venafro, Edizioni Eva, 2004, pp. 17-41, in cui si ribadisce come questo culto sia comunque originario dell'area laziale, con rimandi bibliografici alla tradizione di studi su questa complessa figura agiografica.

${ }^{32}$ L. Allodi \& G. Levi (a cura di), Il Regesto sublacense del secolo XI, Roma, Società Romana di Storia Patria, 1885, doc. n.17, p. 49, a. 936; nella conferma dei beni dell'Abbazia nella città di Anagni si cita un possedimento prossimo ad un oratorio dedicato a S. Secondina: 'atque clusura super se vineata in integra intro civitate vetere anagniensis iuxta sancta secundina'.

33 Lectionarium per annum, cit., ff. 199-200.

34 È importante sottolineare l'importanza in chiave politica di una figura come Magno per la sua diffusa devozione in area pugliese, nel momento storico di progressivo avvicinamento di Roma all'aristocrazia normanna, culminato nella figura di Vittore III, per cui si veda H. E. J. Cowdrey, L'abate Desiderio e lo splendore di Montecassino, Milano, J aca Book, 1985, pp. 173-251.

${ }^{35} \mathrm{~V}$. Fenicchia, 'Intorno agli Atti di san Pietro da Salerno, vescovo di Anagni nel secolo XI, contenuti nel Cod. Chigiano C. VIII. 235', in: Archivio della Regia Deputazione Romana di Storia Patria, 67 (1944), pp. 253-267.

${ }^{36}$ Grégoire, 'La memoria agiografica', cit., p. 20.
} 
l'ambito locale al punto da non poter essere taciute, anzi ribaltate ad offrire un altro aspetto fondativo della santità del vescovo salernitano. ${ }^{37}$

In questo senso ci si deve chiedere se tali contestazioni non tradiscano l'esistenza di una fazione avversa a Pietro ovvero ostile al partito riformista, al quale evidentemente Pietro apparteneva, 0 più semplicemente restia ad accettare l'imposizione di un vescovo esterno. Ciò deve aver comportato il bisogno da parte del vescovo di rafforzarsi nella sua posizione, attraverso la realizzazione di un programma politico e 'propagandistico' come quello che si è descritto.

II modo di rappresentare il suo potere, per Pietro, è fatto di un insieme di elementi che appaiono evidenti oggi, ma probabilmente dovevano essere percepiti come tali anche allora. Una rappresentazione che non poteva essere fine a se stessa, ma rivolta all'esterno, all'osservatore che non doveva essere soltanto la comunità anagnina per quanto estesa potesse essere la diocesi in quel tempo. ${ }^{38}$ In questo ambito locale il suo portfolio di rimandi faceva di Anagni nel pieno medioevo una diocesi aspirante a rivestire il ruolo di centro egemone nella regione, ed essere idealmente ma anche concretamente un punto di riferimento nell'assetto del fitto sistema diocesano del comprensorio ernico. L'insieme dei richiami messi in campi dal vescovo dovevano rappresentare l'importanza, anche politica, della sua sede diocesana agli occhi di un congresso più ampio, cioè il palcoscenico della Roma del papato riformatore, quello in cui determinate rappresentazioni potevano essere percepite anche a livello politico nella loro dimensione storica.

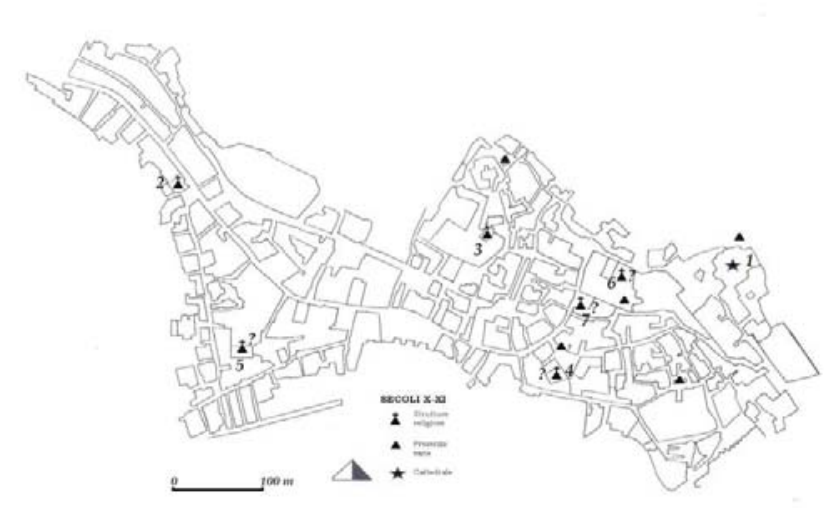

Fig. 1 Elaborazione dell'autore su base cartografica in M. Mazzolani, Anagnia, Roma 1966. Carta topografica di Anagni: 1) Cattedrale di S. Maria; 2) Chiesa di S. Andrea; 3) Chiesa di S. Pancrazio; 4) Chiesa di S. Giovanni de Duce; 5) Chiesa di S. Angelo; 6) Chiesa dei SS. Cosma e Damiano; 7) Chiesa di S. Michele.

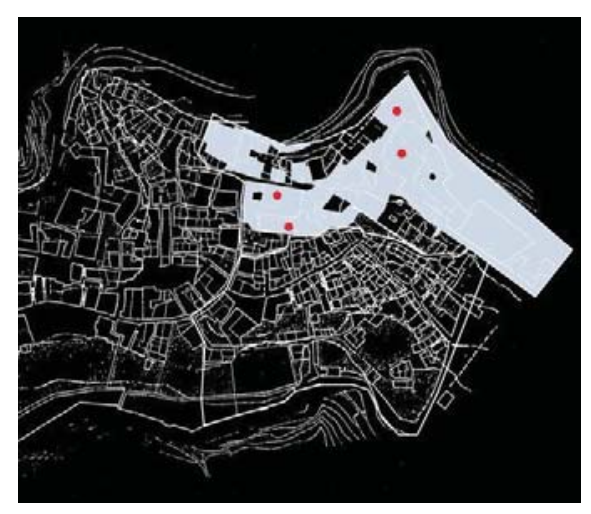

Fig. 2 Elaborazione dell'autore su base cartografica in P. Carlotti Spazio e cultura ad Anagni: la strada Pozzo della valle, Firenze 1998. Stralcio della carta topografica di Anagni con indicazione del complesso episcopale in età tardo medievale, con indicazioni evidenziate dei luoghi dove sono attestate presenze storiche di epoca romanica.

\footnotetext{
${ }^{37}$ Fenicchia, 'Intorno agli Atti di san Pietro da Salerno', cit., p. 261.

${ }^{38} \mathrm{Cfr}$. I. Herklotz, Gli eredi di Costantino. Il papato, il Laterano e la propaganda visiva nel XII secolo, Roma, Viella, 2000, pp. 12-13, le scelte in chiave propagandistica del papato erano rivolte a tutti i potenziali spettatori, soprattutto quando in gioco c'era l'accrescimento della potenza del papato stesso, ed è implicito che questo si dovesse riflettere anche a livello locale.
} 


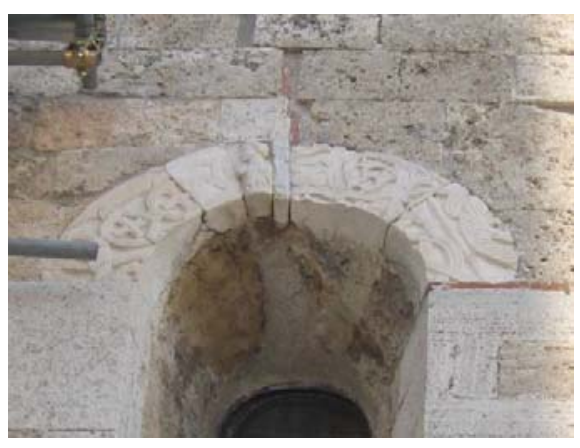

Fig. 3 (c) 2013, Cristiano Mengarelli. Cattedrale di S. Maria, fianco ovest, ghiera d'arco con materiale scultoreo di epoca romana ed alto medievale riutilizzato.

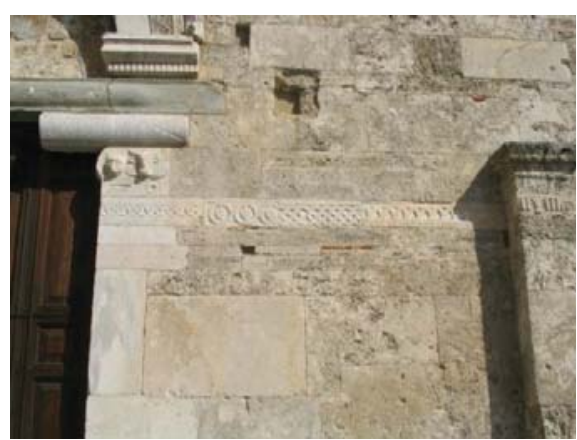

Fig. 5 (c) 2013, Cristiano Mengarelli. Cattedrale di S. Maria, facciata, elementi scultorei riutilizzati, lato destro.

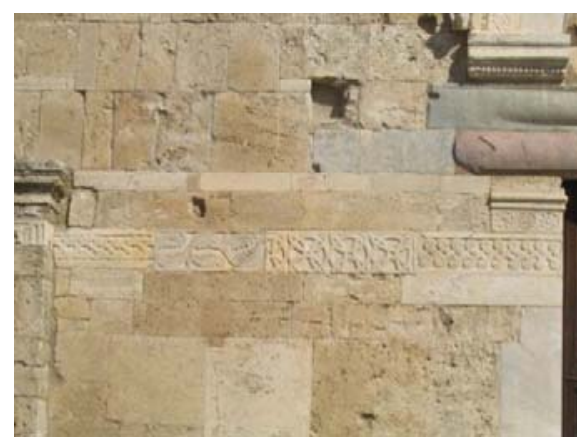

Fig. 4 (c) 2013, Cristiano Mengarelli. Cattedrale di S. Maria, facciata, elementi scultorei riutilizzati, lato sinistro.

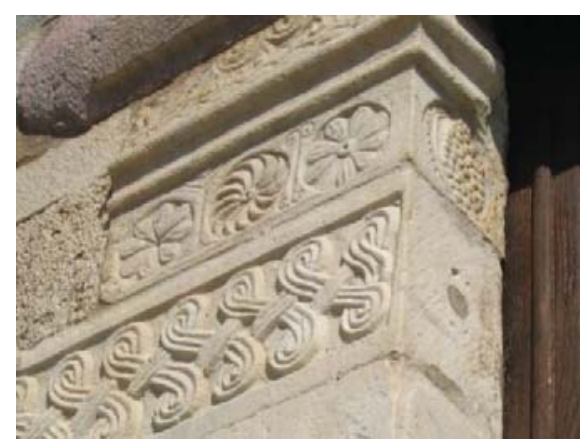

Fig. 6 (c) 2013, Cristiano Mengarelli. Cattedrale di S. Maria, facciata, dettaglio dello spessore in murature degli elementi scultorei riutilizzati. 


\section{Parole chiave}

Anagni, Pietro da Salerno, cattedrale romanica, vescovo, Salerno

Cristiano Mengarelli lavora come archeologo collaboratore della Soprintendenza archeologica per il Lazio. Nel 2009 si è dottorato presso l'Università di Roma Sapienza con una tesi sulle 'Scelte insediative ed organizzazione urbanistica nelle città del comprensorio dei Monti Ernici dalla tarda antichità al medioevo'. Gli interessi di studio si muovono verso ricerche orientate alla comprensione dei processi evolutivi degli insediamenti storici soprattutto dal punto di vista topografico con particolare riguardo al periodo alto medievale.

Via dei Bandinelli 24

00163, Roma (Italia)

cristianomengarelli@gmail.com

\section{SUMMARY}

\section{Representation of Power}

The Bishop of Anagni, Pietro da Salerno († 1105)

In the history of Anagni, Bishop Pietro da Salerno was fundamental in guaranteeing that the city played an important political role throughout the Middle Ages. His work as a spiritual guide, his interventions on the urban layout and the way in which this combination of factors was reflected in the historical memories which formed around the figure of the bishop help to consolidate its historical tradition. Pietro da Salerno used his office of bishop in the certainty that he was acting on behalf of an established power, to be consolidated in his own time and offered as a stable and secure legacy for the future. The representation of this power took the form of an action program, centered around the urban renewal of an entire city district with the aim of creating an architectural 'macro-palimpsest' serving as a political manifesto based on the recovery, through continuity, of the historical, hagiographic and material legacy which defined and recalled, in a simple but immediate form, the power of the bishops over the city of Anagni. We are thus dealing with a legitimization of power through the representation of historical events and allusions, expressed through the stylistic features of the contemporary cultural tradition. 